\title{
Editorial
}

\section{Variational Analysis, Optimization, and Fixed Point Theory 2014}

\author{
Jen-Chih Yao, ${ }^{1}$ Abdul Latif, ${ }^{2}$ Chong Li, ${ }^{3}$ and Adrian Petrusel ${ }^{4}$ \\ ${ }^{1}$ Center of General Education, Kaohsiung Medical University, Kaohsiung 807, Taiwan \\ ${ }^{2}$ Department of Mathematics, King Abdulaziz University, Jeddah 21589, Saudi Arabia \\ ${ }^{3}$ Department of Mathematics, Zhejiang University, Hangzhou 3110027, China \\ ${ }^{4}$ Department of Mathematics, Babes-Bolyai University, 400084 Cluj-Napoca, Romania
}

Correspondence should be addressed to Jen-Chih Yao; yaojc@kmu.edu.tw

Received 14 September 2014; Accepted 14 September 2014

Copyright ( $) 2015$ Jen-Chih Yao et al. This is an open access article distributed under the Creative Commons Attribution License, which permits unrestricted use, distribution, and reproduction in any medium, provided the original work is properly cited.

In the last two decades, the theory of variational analysis including variational inequalities (VI) emerged as a rapidly growing area of research because of its applications in nonlinear analysis, optimization, economics, game theory, and so forth; see, for example, [1] and the references therein. In the recent past, many authors devoted their attention to studying the VI defined on the set of fixed points of a mapping, called hierarchical variational inequalities. Very recently, several iterative methods have been investigated to solve VI, hierarchical variational inequalities, and triple hierarchical variational inequalities. Since the origin of the VI, a tool has been used to study optimization problems. Hierarchical variational inequalities are used to study the bilevel mathematical programming problems. A triple level mathematical programming problem can be studied by using triple hierarchical variational inequalities. Several abstract results in nonlinear analysis are of special interest and applicability in the theory of variational problems, optimization, and mathematical economics. We point out here three of them (we refer to [2] for other methods or approaches).

Ekeland's variational principle provides the existence of an approximate minimizer of a bounded below and lower semicontinuous function. It is one of the most important results from nonlinear analysis and it has applications in different areas of mathematics and mathematical sciences, namely, fixed point theory, optimization, optimal control theory, game theory, nonlinear equations, dynamical systems, and so forth, for example, [3-8] and the references therein.
During the last decade, it has been used to study the existence of solutions of equilibrium problems in the setting of metric spaces, for example, $[3,4]$ and the references therein.

Banach's contraction principle is remarkable in its simplicity, yet it is perhaps the most widely applied fixed point theory in all of the analyses. This is because the contractive condition on the mapping is simple and easy to verify and because it requires only completeness of the metric space. Although, the basic idea was known to others earlier, the principle first appeared in explicit form in Banach's 1922 thesis where it was used to establish the existence of a solution to an integral equation.

Caristi's fixed point theorem $[9,10]$ has found many applications in nonlinear analysis. It is shown, for example, that this theorem yields essentially all the known inwardness results of geometric fixed point theory in Banach spaces. Recall that inwardness conditions are the ones which assert that, in some sense, points from the domain are mapped toward the domain. This theorem is an amazing equivalent to Ekeland's variational principle. We refer to the recent monograph [11].

This special issue is concerned with the most recent development on the topic.
Jen-Chih Yao

Abdul Latif

Chong $\mathrm{Li}$

Adrian Petrusel 


\section{References}

[1] Q. H. Ansari, C. S. Lalitha, and M. Mehta, Generalized Convexity, Nonsmooth Variational Inequalities, and Nonsmooth Optimization, CRC Press, Taylor \& Francis, Boca Raton, Fla, USA, 2014.

[2] C. Border Kim, Fixed Point Theorems with Applications to Economics and Game Theory, Cambridge University Press, Cambridge, UK, 1989.

[3] Q. H. Ansari, Metric Spaces: Including Fixed Point Theory and Set-Valued Maps, Narosa Publishing House, New Delhi, India, 2010.

[4] M. Bianchi, G. Kassay, and R. Pini, "Existence of equilibria via Ekeland's principle," Journal of Mathematical Analysis and Applications, vol. 305, no. 2, pp. 502-512, 2005.

[5] D. G. De Figueiredo, The Ekeland Variational Principle with Applications and Detours, Tata Institute of Fundamental Research, Mumbai, India, 1989.

[6] I. Ekeland, "Sur les prolèms variationnels," Comptes Rendus de l'Académie des Sciences, vol. 275, pp. 1057-1059, 1972.

[7] I. Ekeland, "On the variational principle," Journal of Mathematical Analysis and Applications, vol. 47, pp. 324-353, 1974.

[8] I. Ekeland, "Nonconvex minimization problems," The American Mathematical Society. Bulletin, vol. 1, no. 3, pp. 445-474, 1979.

[9] J. Caristi, "Fixed point theorems for mappings satisfying inwardness conditions," Transactions of the American Mathematical Society, vol. 215, pp. 241-251, 1976.

[10] J. Caristi and W. A. Kirk, "Geometric fixed point theory and inwardness conditions," in The Geometry of Metric and Linear Spaces, vol. 490 of Lecture Notes in Mathematics, pp. 74-83, Springer, Berlin, Germany, 1975.

[11] M. A. Khamsi and W. A. Kirk, An Introdunction toMetric Spaces and Fixed Point Theory, Wiley, New York, NY, USA, 2001. 


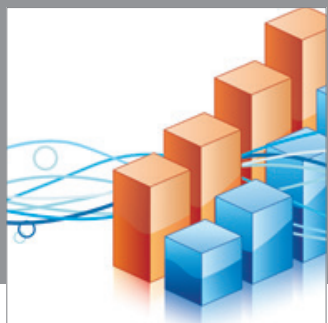

Advances in

Operations Research

mansans

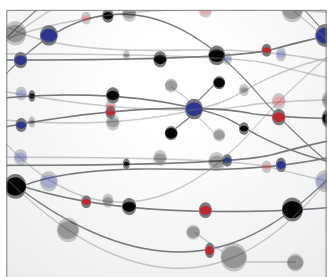

The Scientific World Journal
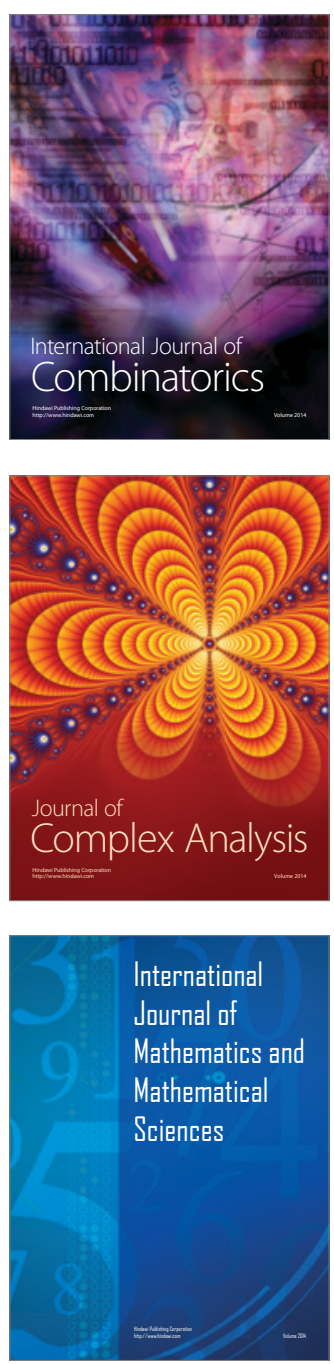
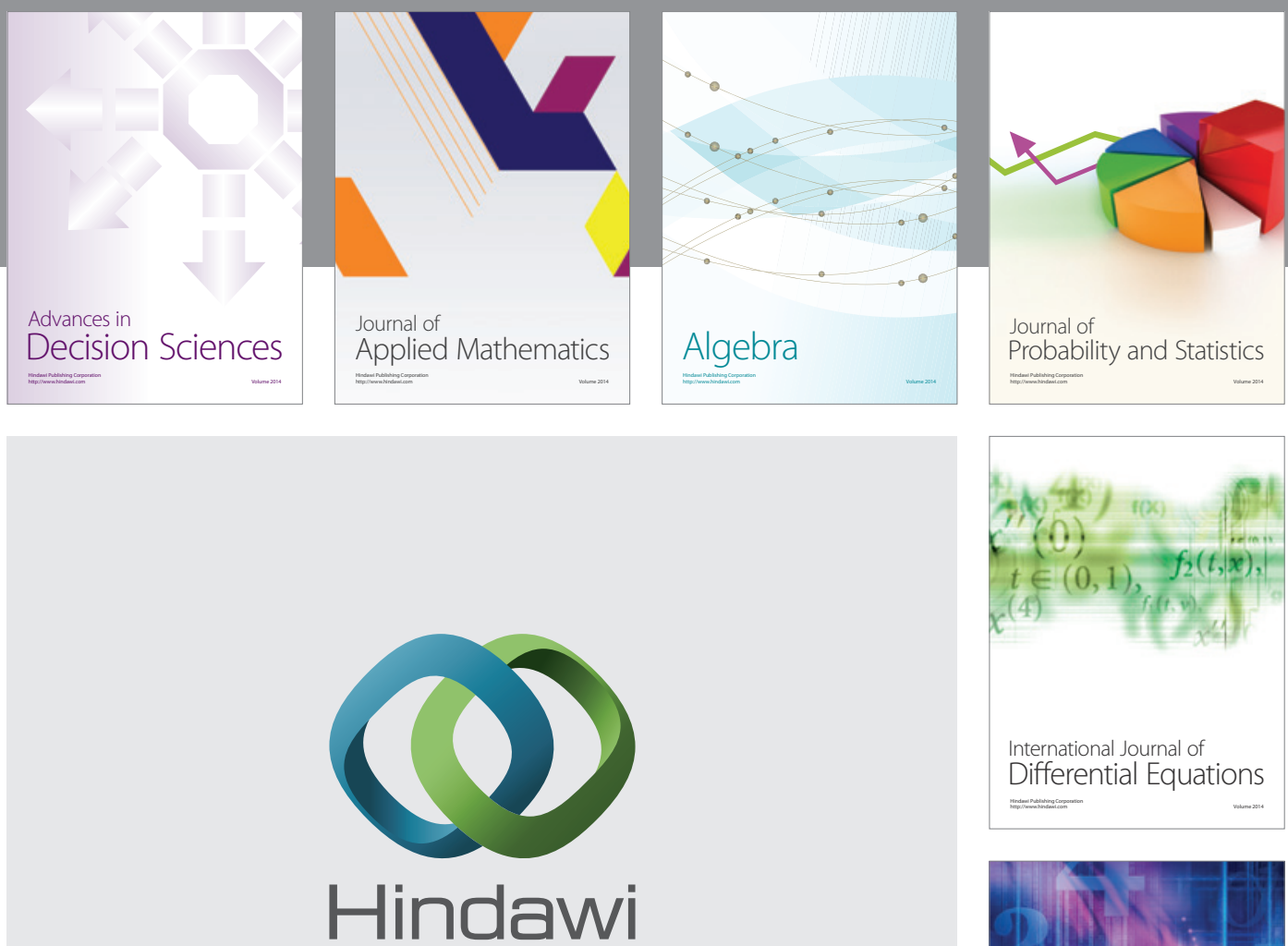

Submit your manuscripts at http://www.hindawi.com
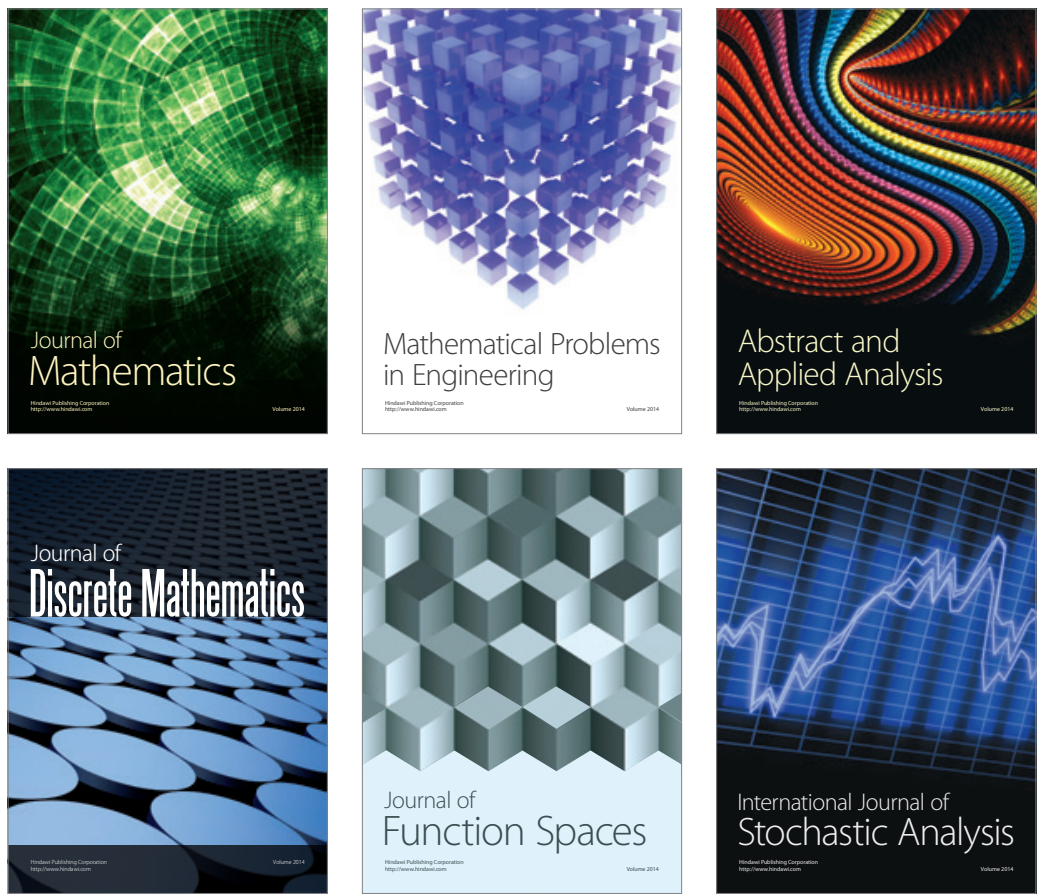

Journal of

Function Spaces

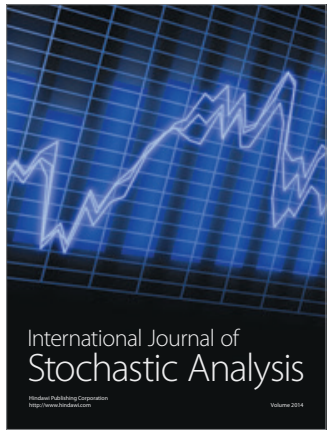

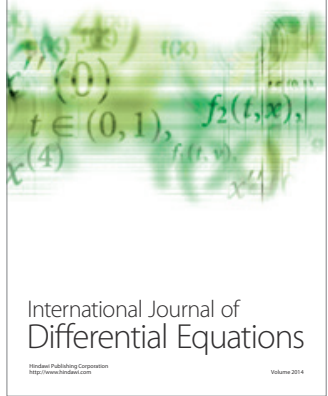
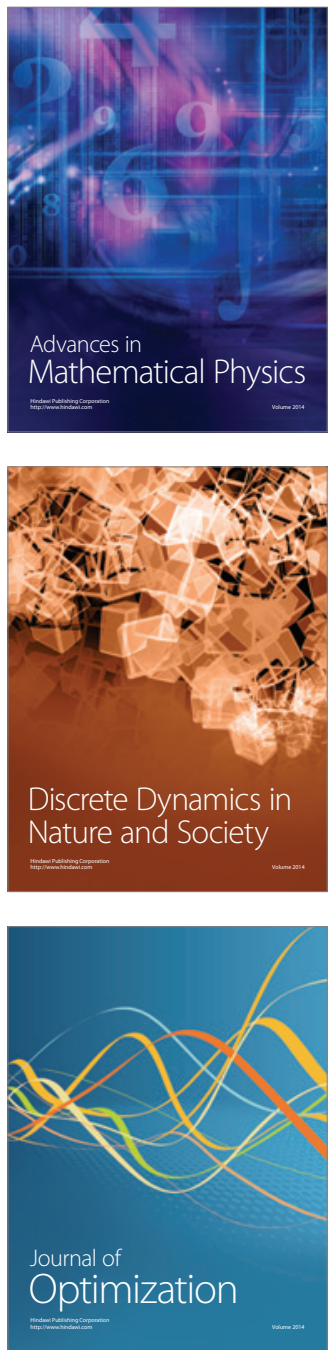